\title{
Imaging gene-substance interactions: The effect of the DRD2 TaqIA polymorphism and the dopamine agonist bromocriptine on the brain activation during the anticipation of reward
}

\author{
Peter Kirsch $^{\mathrm{a}, *}$, Martin Reuter $^{\mathrm{b}}$, Daniela Mier ${ }^{\mathrm{a}}$, Tina Lonsdorf ${ }^{\mathrm{b}}$, \\ Rudolf Stark $^{\mathrm{b}, \mathrm{c}}$, Bernd Gallhofer ${ }^{\mathrm{a}}$, Dieter Vaitl ${ }^{\mathrm{c}}$, Jürgen Hennig ${ }^{\mathrm{b}}$ \\ ${ }^{a}$ Center for Psychiatry, Justus-Liebig-University Giessen, Am Steg 28, D-35385 Giessen, Germany \\ ${ }^{\mathrm{b}}$ Department of Psychology, Justus-Liebig-University Giessen, Giessen, Germany \\ ${ }^{\mathrm{c}}$ Bender Institute of Neuroimaging, Justus-Liebig-University Giessen, Giessen, Germany
}

\begin{abstract}
Dopamine is known as the main neurotransmitter modulating the activation of the reward system of the brain. The DRD2 TaqIA polymorphism is associated with dopamine D2 receptor density which plays an important role in the context of reward. Persons carrying an A1 allele have a lower D2 receptor density and a higher risk to show substance abuse. The present study was designed to investigate the influence of the DRD2 TaqIA polymorphism and the selective D2 receptor agonist bromociptine on the activation of the reward system by means of functional magnetic resonance imaging (fMRI). In a double-blind crossover study with 24 participants we found an increase of reward system activation from placebo to bromocriptine only in subjects carrying the A1 allele. Furthermore, only A1 carrier showed an increase of performance under bromocriptine. The results are interpreted as reflecting a specific sensitivity for dopamine agonists in persons carrying an A1 allele and may complement actual data and theories of the development of addiction disorders postulating a higher genetic risk for substance abuse in carrier of the A1 allele.
\end{abstract}

Keywords: Dopamine; DRD2; Reward; Addiction; fMRI; Genetics

The dopaminergic reward system including the ventral striatum as one core structure is sensitive for the anticipation of a forthcoming reward $[7,6]$. The sensitivity of the reward system seems to be associated with a polymorphism of the dopamine D2 receptor gene (DRD2 TaqIA). Although located on the intron of the gene, the DRD2 TaqIA polymorphisms was found to be associated with the dopamine receptor density, probably via an association with a functional polymorphism in an adjacent gene. The minor A1 allele was found to be associated with a reduced number of dopamine binding sites [5] and was repeatedly [10,12,2] but not always [4] described to be associated with an increased likelihood to develop substance abuse and addiction. The relationship between the A1 allele and addiction was often linked to the so-called "reward deficiency syndrome" $[1,2]$. It has been argued for a long time, that dopamine that is released

\footnotetext{
* Corresponding author. Tel.: +49 641 9946774; fax: +49 6419945789. E-mail address: peter.kirsch@psychiat.med.uni-giessen.de (P. Kirsch).
}

from the ventral striatum after consuming substances like alcohol or drugs serves as a reward signal in the brain. Therefore, since persons who carry the A1 allele show a reduced sensitivity to reward, they feel the increased urge to consume substances of abuse stimulating the dopamine (DA) system. This phenomenon is described as craving. Lawford et al. [8] showed that craving behaviour in alcohol abusers carrying the A1 allele can be reduced by the dopamine agonist bromocriptine.

However, it has been shown that dopamine neurons do not only respond to the delivery of a reward but also to the presentation of a reward-predicting stimulus [16]. Therefore, if the A1 allele of the DRD2 TaqIA gene reduces the sensitivity of the reward system, one would expect, that this reward deficiency syndrome should also influence the activation of the reward system during the anticipation of reward. Tran et al. [17] found reduced pre-reward activity in the nucleus accumbens (NAcc) of DRD2 knock out mice in an operant spatial learning task. Findings are interpreted as an indicator for the important role of D2 receptors in the NAcc for coding an expected reward. On the 
other hand, providing a dopamine agonist to an A1 allele carrier might especially increase the sensitivity of the reward system during the anticipation of future rewards leading to increased reward seeking behaviour.

The present study was carried out in order to further investigate the influence of these relations between genetic disposition and dopamine agonists on the activity of the human reward system during the anticipation of a reward by means of functional magnetic resonance imaging (fMRI). We presented a reward anticipation paradigm that was shown to effectively activate structures of the reward system like the ventral striatum [6].

We expected a main effect of the substance on the activation of the core region of the reward system, the ventral striatum during the anticipation of a monetary reward. Furthermore, we expected an interaction between DRD2 type and substance with a stronger increase of reward system activation during reward anticipation from placebo to bromocriptine in those participants who carry at least one A1 allele.

Participants were 24 healthy Caucasian volunteers of German origin who gave informed consent before participation. The study protocol was in accordance with the Declaration of Helsinki.

In advance it was tested that all participants were free of present or past physical or psychiatric illness. Subjects were 12 males and 12 females. The mean age of the participants was 25.7 years (range 19-37). Out of a gene data bank consisting of more than 700 healthy Caucasians, the participants were recruited with respect to their DRD2 genotype. Eight subjects had a A1A1 genotype, eight subjects a A1A2 genotype and eight subjects had a A2A2 genotype.

The participants were investigated in two sessions with an interval of 1 week. They were paid $€ 80$ for their participation. Additional money was gained during the reward experiment.

DNA was extracted from buccal cells to avoid a selective exclusion of subjects with blood and injection phobias. Purifica- tion of genomic DNA was performed with a standard commercial extraction kit (High Pure PCR Template Preparation Kit; Roche Diagnostics, Mannheim, Germany). Genotyping of the four single nucleotide polymorphisms (SNPs) was performed by real-time PCR using fluorescence melting curve detection analysis by means of the Light Cycler System (Roche Diagnostics). Details of the PCR protocols were described elsewhere $[15,13,11]$. The primers and hybridization probes used (TIB MOLBIOL, Berlin, Germany) for the analysis of the DRD2 TaqIA SNP were as follows:

forward primer: 5'-CGGCTGGCCAAGTTGTCTAA-3'; reverse primer: 5'-CAAATGTCCACGCCCGCA-3'; anchor hybridization probe: $5^{\prime}$-LCRed640-TGAGGATGGCTGTGTTGCCCTT-phosphate- $3^{\prime}$; sensor hybridization probe: 5'-CTGCCTCGACCAGCACTfluorescein- $3^{\prime}$.

Two hours in advance of the fMRI testing an oral dose of $1.5 \mathrm{mg}$ bromocriptine or placebo packed in an identical capsule were administered with a glass of milk to prevent nausea. The participants were not able to differentiate verum from placebo. Only one participant experienced nausea as a side effect but this occurred after the scanning session. Participants were seated in a comfortable armchair and were allowed to read magazines while waiting for the expected maximal plasma concentrations $\left(T_{\max }\right)$ of the drug. At $T_{\max }$ subjects were tested in the scanner.

During scanning, the subjects were presented four different conditions (Fig. 1). The first condition, the so-called monetary reward condition, was initiated by a vertically oriented arrow with a head on top. It was presented for $6 \mathrm{~s}$ and immediately followed by the task (a flashlight as imperative stimulus) without any inter stimulus interval. After the subjects had responded to the flash by pressing a response button as quick as possible, a feedback was given whether or not they had responded fast



Fig. 1. The four experimental conditions presented in the experiment. Participants were instructed to respond to a flash that was presented following the presentation of the CS using a response button. CS presentation was $6 \mathrm{~s}$ for all conditions. 
enough to earn money $(€ 2)$. In the second condition, the socalled punishment avoidance condition, the initial stimulus was a vertically oriented arrow pointing downwards. Again, the task was presented $6 \mathrm{~s}$ after trial onset. However, in this case a slow response was punished by a loss of $€ 2$ while a fast response was rewarded by the avoidance of that loss. Interestingly, the activation pattern of the first two conditions did not differ substantially. Therefore, we report only data from the monetary reward condition in this paper.

The third condition, called the verbal feedback condition, consisted of a vertically oriented arrow with heads on both ends. The only difference to the two monetary conditions was that the feedback contained no information about a monetary gain, only information was given whether the response was fast or slow.

For all of these three conditions, the threshold for a fast response was set adaptive for each subject and each trial to ensure that all subjects: (1) were able to win some money and (2) work on their maximum performance level. The adaptive algorithm was a simple increase of $5 \%$ of the threshold after a slow response and a $10 \%$ decrease after a fast response.

In another control condition, a horizontally oriented arrow with a head on both ends was presented for $6 \mathrm{~s}$. This stimulus was followed by a black screen for $3 \mathrm{~s}$. As usual in differential conditioning paradigms, this condition was used to include a control condition without any anticipation of a consequence. Two seconds after each task the actual account balance was displayed for $2 \mathrm{~s}$. The inter-trial interval was randomly varied between 6 and 9 s. Each condition was presented 20 times in a pseudo random trial order with not more than 2 equal conditions in succession.

The task was presented using the Presentation software package (Neurobehavioral Systems, Albany, CA). The stimuli were presented with a LCD projector on a screen on the backside of the scanner. The subjects were able to watch the screen, using a mirror located approximately $20 \mathrm{~cm}$ above their eyes.

Functional imaging data were acquired by a $1.5 \mathrm{~T}$ Siemens Symphony whole body MRI-scanner with a Quantum gradient system (Siemens, Erlangen, Germany). In order to measure the blood oxygenation level dependent (BOLD) contrast, a T2* weighted single shot gradient echo EPI sequence $(\mathrm{TR}=3 \mathrm{~s}$, $\mathrm{TE}=55 \mathrm{~ms}$, flip angle $=90^{\circ}, \mathrm{FOV}=192 \mathrm{~mm}, 64 \times 64$ matrix $)$ was used. One volume contained 30 slices with $4 \mathrm{~mm}$ slice thickness with $1 \mathrm{~mm}$ gap and covered the whole brain. The slices were acquired in descending order. Prior to the functional measurement, a T1-weighted anatomical MRI scan was acquired for each subject.

The fMRI data were analyzed using statistical parametric mapping methods with the SPM2 software package (The Wellcome Department of Cognitive Neurology, London, England). During preprocessing, the EPI images were corrected for sequential slice timing. In a next step all images were realigned to the first image to adjust them for head movements. The realigned images were then spatially normalized to a standard EPI template to allow averaging across subjects. In the last preprocessing step, all functional images were smoothed by using a $6 \mathrm{~mm}$ (full width at half maximum) isotropic Gaussian kernel.

The evoked BOLD responses were modelled for the four stimulus conditions as well as the task conditions (task with reward, task without reward, no task). A synthetic hemodynamic response function was used for response modelling. In order to account for movement related variance that might be correlated to the experimental design, the six movement parameters (three translations and three rotations) that were derived from the realignment preprocessing step, were included as covariates into the analysis. In order to test differences between conditions, linear contrasts were applied to the estimates of the parameters for each condition. Furthermore, interactions between task conditions and substance conditions were tested on the first level separately for each subject. In a second step, the contrast images of each subject were then included into a random-effects second level analysis. For the whole group analyses, the resulting $p$-values of the $t$-statistic of the specific contrasts were adjusted for multiple comparisons within the entire volume using the Gaussian random field theory (family wise error correction). For the group comparisons, region of interest analyses were conducted with the left and right nucleus accumbens as target region. The required masks for these analyses were designed using the software-program MARINA (http://www.bion.de/Marina.htm). The masked consisted of 154 voxels $\left(4158 \mathrm{~mm}^{3}\right)$ for the right side mask and 198 voxels $\left(5348 \mathrm{~mm}^{3}\right)$ for the left side mask. For the ROI analyses, the resulting $p$-values of the $t$-statistic were adjusted for multiple comparisons within the ROI, again using family wise error correction.

Performance was analyzed in terms of reaction time as well as money gained during the task. To make both measures comparable, the reaction times were computed as difference between reaction times in the unrewarded task minus reaction times in the rewarded task. Due to the relatively small number of subjects, comparing the three genotype groups did not reveal a significant main effect. Therefore, to increase power, we compared subjects who carried at least one A1 allele (A1+) with those, who were homozygote for the A2 allele (A1-). This kind of grouping has been done in a number of studies on the DRD2 TaqIA polymorphism (see [10]).

With respect to the monetary gain, a significant genotype main effect occurred $(F(1 / 21)=5.04, p<.05)$ with more money obtained by the A $1-$ group. Furthermore, we found a significant interaction between genotype and substance $(F(1 / 21)=5.23$, $p<.05)$ with an significant difference between $\mathrm{A} 1+$ and $\mathrm{A} 1-$ only in the placebo condition (Fig. 2a). The same pattern of results occurred with regards to the reaction times, however, due to a larger variance, the effects showed only a trend towards significance (main effect: $F(1 / 21)=3.53, p<.08$; interaction effect: $F(1 / 21)=3.31, p<.09$; Fig. $2 b)$.

There were no performance differences between females and males.

The analyses of brain activation revealed a significant effect of monetary reward: during the anticipation of monetary reward the structures of the brain reward system were significantly more activated than during the anticipation of verbal reward (Fig. 3a and Table 1). However, no main effect for genotype, substance or gender could be observed, neither for the whole brain nor for the ROI analyses. For the investigation of the interaction between task condition, substance and genotype, a second level one-way ANOVA with the DRD2 type as group 

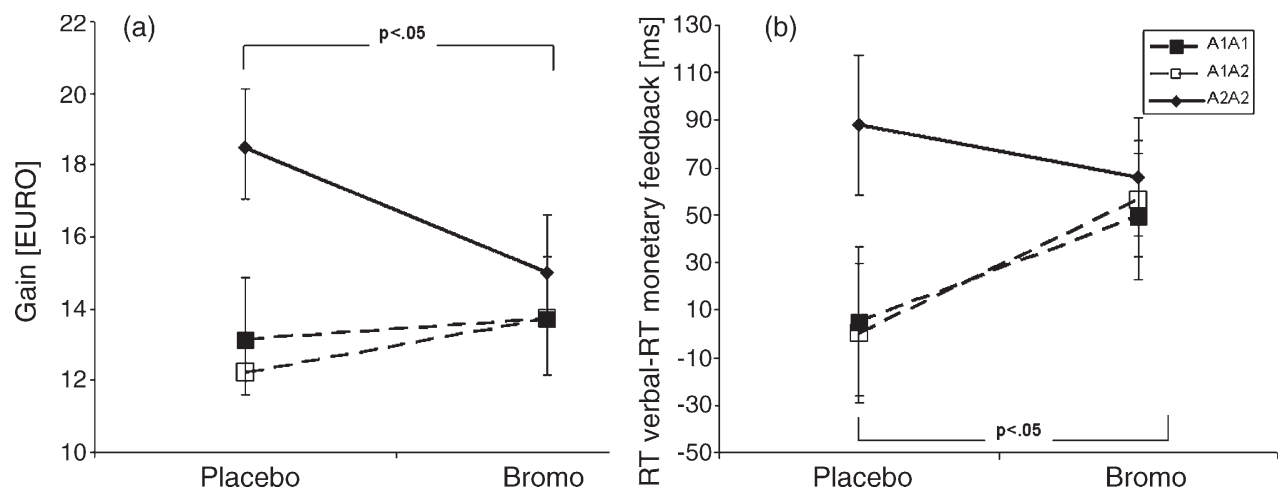

Fig. 2. Influence of substance and genotype on the performance in the reward anticipation task. (a) Money obtained by the subjects ( $M$ and S.E.M.). (b) Amount of reaction time reduction during monetary compared to verbal conditions ( $M$ and S.E.M.). $p$-Values are from post hoc $t$-test (Bonferroni corrected).

factor was performed. When analysing the increase of brain activation from placebo to bromocriptine for the contrast monetary reward minus verbal feedback, significantly more activation was found in A1 allele carriers, specifically limited to the right NAcc (peak activation-Montreal Neurological Institute (MNI)-coordinates: $x=15, y=9, z=-15, T(21)=4.3, p$ (ROIcorrected $)=.016$; Fig. $3 \mathrm{~b}$ ). This three-way interaction between task condition, substance and genotype gives clear evidence for an increase of reward system activation in the A1A1 participants under dopamine agonistic influence. Furthermore, this effect was observed as linear contrast for the three DRD2 groups, with the highest response under bromocriptine to the monetary condition and under placebo to the verbal condition in the A1A1 and the lowest responses in the A2A2 group (Fig. 3c).
In the present study, the influence of the DRD2 genotype and the dopamine agonist bromocriptine on the reward system activation was investigated with respect to performance and brain activation data.

For the performance measures, a significant influence of the DRD2 genotype on the amount of money gained as well as the increase of reaction time under motivational conditions was observed. A1+ participants were less effective in gaining money and showed a reduced improvement of performance under high incentive conditions. However, this effect occurred only under placebo conditions. Under bromocriptine influence, the performance deficit was abolished (Fig. 2). The results can be interpreted as reflecting the reward deficiency syndrome in the A1+ group which is reduced under the influence of a dopamine ago-
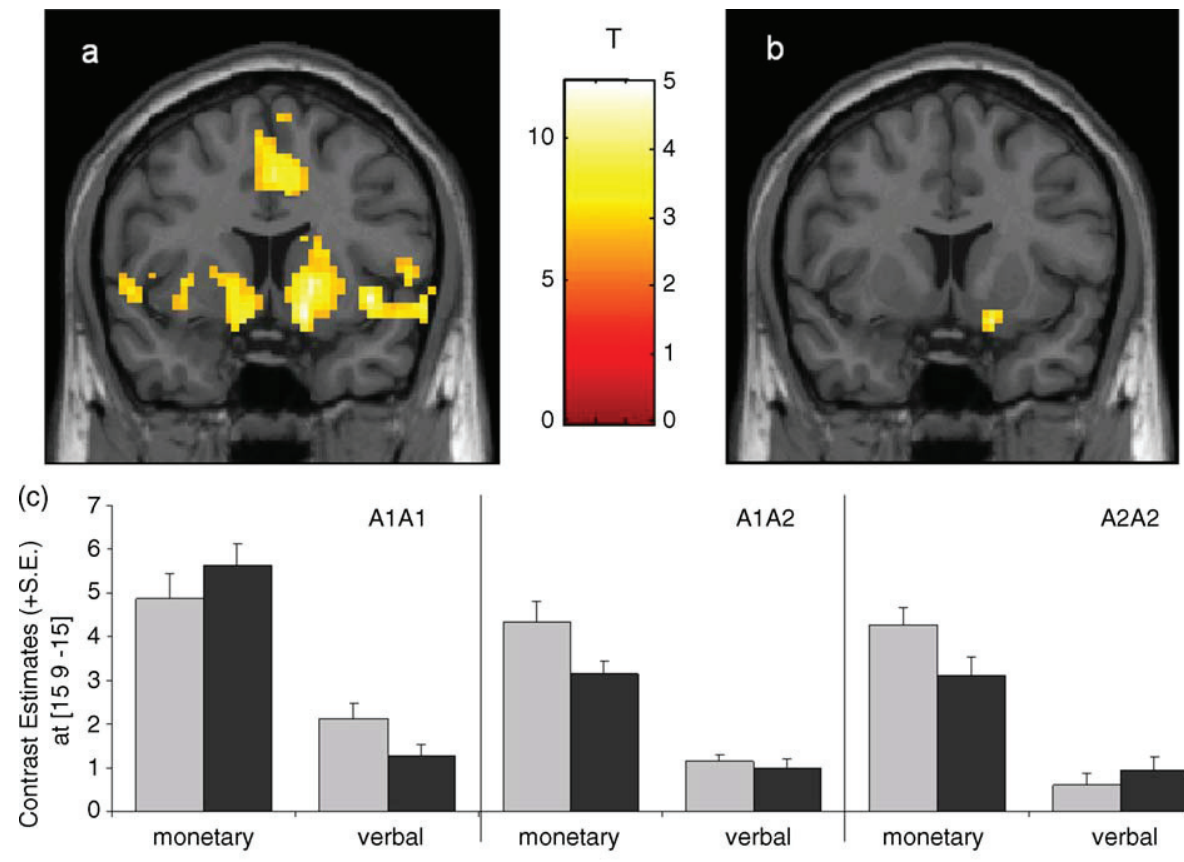

$\mathrm{A} 1 \mathrm{~A} 2$

A2A2

$\square$ Placebo

Bromocriptin

Fig. 3. Increased activation of the striatum during the anticipation of reward rendered on a normal coronal MRI at $y=9$ (MNI-space). Displayed are results from the contrast monetary reward $>$ verbal feedback. (a) Main effect for all subjects and both substance conditions ( $p<.05$, corrected for the entire brain). (b) Stronger increase of activation in the bromocriptine as in the placebo condition depended on the genotype (A1A1 $>$ A1A2 $>$ A2A2, $p<.05$ corrected for the ROI). Left $T$ scale corresponds to (a), and right to (b). (c) Means (+standard errors) of the contrasts for the monetary reward and verbal feedback conditions under placebo and bromocriptine, separated for the three genotype groups in the right NAcc. 
Table 1

Locations and $T$-values for the significant activations for the contrast monetary > verbal feedback for all participants and both substances

\begin{tabular}{|c|c|c|c|c|c|}
\hline \multirow[t]{2}{*}{ Area (Talairach Label/Brodman's Area) } & \multicolumn{3}{|c|}{ MNI coordinates } & \multirow[t]{2}{*}{$T_{\max }$} & \multirow[t]{2}{*}{$p_{\text {corrected }}$} \\
\hline & $x$ & $y$ & $z$ & & \\
\hline Left nucleus accumbens & -12 & 9 & -15 & 9.14 & .0002 \\
\hline Right nucleus accumbens & 12 & 9 & -12 & 11.23 & .000003 \\
\hline Left thalamus & -9 & -15 & 3 & 8.50 & .0005 \\
\hline Right thalamus & 6 & -21 & 3 & 7.16 & .01 \\
\hline Left amygdala & -21 & 0 & -12 & 9.04 & .0003 \\
\hline Right amygdala & 27 & -6 & -12 & 8.66 & .0004 \\
\hline Left putamen & -21 & 0 & 3 & 9.62 & .0001 \\
\hline Right putamen & 15 & 12 & -6 & 9.93 & .00003 \\
\hline Left orbitofrontal cortex (BA 47) & -30 & 30 & -9 & 7.21 & .008 \\
\hline Right orbitofrontal cortex (BA 47) & 51 & 21 & -15 & 6.42 & .03 \\
\hline Left cingulate gyrus (BA 32) & -6 & 12 & 36 & 7.35 & .007 \\
\hline Right cingulate gyrus (BA 24) & 6 & 24 & 27 & 9.06 & .0002 \\
\hline Left insula (BA 13) & -45 & 3 & 3 & 10.01 & .00003 \\
\hline Right insula (BA 13) & 39 & 12 & -6 & 11.17 & .000004 \\
\hline Right substantia nigra & 12 & -21 & -9 & 6.97 & .02 \\
\hline Left precentral gyrus (BA 4) & -36 & -18 & 51 & 9.92 & .00003 \\
\hline Right precentral gyrus (BA 6) & 51 & -3 & 48 & 7.80 & .003 \\
\hline Right postcentral gyrus (BA 3) & 60 & -15 & 27 & 9.01 & .0003 \\
\hline Left superior temporal gyrus (BA 22) & -57 & 12 & -3 & 8.04 & .002 \\
\hline Right middle temporal gyrus (BA 22) & 54 & -30 & -6 & 7.93 & .002 \\
\hline Left superior parietal lobule (BA 7) & -24 & -51 & 60 & 9.61 & .00006 \\
\hline Left inferior parietal lobule (BA 40) & -60 & -21 & 24 & 7.65 & .004 \\
\hline Left lingual gyrus (BA 19) & -24 & -66 & 3 & 8.93 & .0003 \\
\hline Right superior occipital gyrus (BA 19) & 42 & -75 & 24 & 7.48 & .005 \\
\hline Right precuneus (BA 7) & 9 & -72 & 51 & 8.21 & .001 \\
\hline
\end{tabular}

In the upper part of the table, the most significant voxel within the regions associated with reward are displayed. In the lower part, the most significant voxel of the significantly activated clusters outside these regions are displayed. The $p$-values are family wise error corrected for the entire volume. Coordinates are in the space of the standard brain from the Montreal Neurological Institute (MNI).

nist. Surprisingly, for the A1- group, performance was lower when a dopamine agonist was administered. This finding might indicate an inverted ' $U$ ' shape characteristic between DA activity and reward sensitivity/performance [9], i.e. A1 - subjects without a reward deficiency syndrome, with higher DA receptor density and therefore higher basal DA activity are pushed beyond their optimal DA activation level by the DA agonist, whereas A1+ carriers with low receptor density and low DA activity profit from the stimulation of the DA system by restoring their optimal DA tone by means of bromocriptine.

The functional imaging data again demonstrated the suitability of the paradigm used to activate structures of the reward system during the anticipation of monetary reward (Fig. 3a and Table 1). Furthermore, the fMRI data support the assumption of an interaction between DRD2 genotype and substance since an additional increase of NAcc activation under bromocriptine could only be observed for the A1A1 genotype (Fig. 3b and c). Obviously, the increase of reward system activation under dopamine agonistic influence is positively correlated with the number of A1 alleles since the highest activation of the NAcc during monetary reward anticipation under bromocriptine was found for the A1A1 group and the lowest for the A2A2 group (Fig. 3c).

Our present results are in line with older reports of a reduced craving behaviour in A1+ alcohol abusers after bromocriptine challenge [8]. However, they also expand these findings by giving evidence for the assumption that a reduced D2 receptors density and a resulting reward deficiency syndrome does not only increase craving for a dopmainergic substance. The substance itself selectively increases the sensitivity of the reward system. Therefore, only in A1 carriers, consuming a dopamine agonist specifically increases the probability of drug seeking behaviour which might itself increase the probability to develop an addiction disorder by increasing the salience of a potentially rewarding stimulus in the environment which itself must not be drugs. For example, Dodd et al. [3] reported that Parkinson disease (PD) patients treated with a dopamine agonist were very likely to develop a reversible gambling disorder. Probably, the dopamine agonist increased the reward system sensitivity in these patients leading to an increased risk to develop a gambling 
disorder when confronted with the opportunity to win money. Our results would predict that the probability to develop such a disorder as a PD patient treated with dopamine agonists would depend on the individual DRD2 genotype. This conclusion is strongly supported by recent data from Reuter et al. [14] who found a reduced reward system sensitivity to be linked to pathological gambling.

It can be expected that further studies with other dopamine agonist but also with antagonist might give further insights into the relationships between DRD2 receptor density, reward sensitivity and the risk to develop an addiction disorder.

\section{References}

[1] K. Blum, E.R. Braverman, J.M. Holder, J.F. Lubar, V.J. Monastra, D. Miller, J.O. Lubar, T.J. Chen, D.E. Comings, Reward deficiency syndrome: a biogenetic model for the diagnosis and treatment of impulsive, addictive, and compulsive behaviors, J. Psychoactive Drugs 32 (Suppl. i-iv) (2000) 1-112.

[2] A. Bowirrat, M. Oscar Berman, Relationship between dopaminergic neurotransmission, alcoholism, and reward deficiency syndrome, Am. J. Med. Genet. B Neuropsychiatr. Genet. 132 (2005) 29-37.

[3] M.L. Dodd, K.-J. Klos, J.H. Bower, Y.E. Geda, K.A. Josephs, J.E. Ahlskog, Pathological gambling caused by drugs used to treat Parkinson disease, Arch. Neurol. 62 (2005) 1377-1381.

[4] T. Hallikainen, J. Hietala, J. Kauhanen, T. Pohjalainen, E. Syvalahti, J.T. Salonen, J. Tiihonen, Ethanol consumption and DRD2 gene TaqI a polymorphism among socially drinking males, Am. J. Med. Genet. A 119 (2003) 152-155.

[5] E.G. Jönsson, N.M. Nothen, F. Grunhage, L. Farde, Y. Nakashima, P. Propping, G.C. Sedvall, Polymorphisms in the dopamine D2 receptor gene and their relationships to striatal dopamine receptor density of healthy volunteers, Mol. Psychiatry 4 (1999) 290-296.

[6] P. Kirsch, A. Schienle, R. Stark, G. Sammer, C. Blecker, B. Walter, U. Ott, J. Burkart, D. Vaitl, Anticipation of reward in a nonaversive differential conditioning paradigm and the brain reward system: an eventrelated fMRI study, Neuroimage 20 (2003) 1086-1095.
[7] B. Knutson, C.M. Adams, G.W. Fong, D. Hommer, Anticipation of increasing monetary reward selectively recruits nucleus accumbens, J. Neurosci. 21 (2001) RC159.

[8] B.R. Lawford, R.M. Young, J.A. Rowell, J. Qualichefski, B.H. Fletcher, K. Syndulko, T. Ritchie, E.P. Noble, Bromocriptine in the treatment of alcoholics with the D2 dopamine receptor A1 allele, Nat. Med. 1 (1995) 337-341.

[9] V.S. Mattay, T.E. Goldberg, F. Fera, A.R. Hariri, A. Tessitore, M.F. Egan, B. Kolachana, J.H. Callicott, D.R. Weinberger, Catechol Omethyltransferase val158-met genotype and individual variation in the brain response to amphetamine, Proc. Natl. Acad. Sci. U.S.A. 100 (2003) 6186-6191.

[10] E.P. Noble, Addiction and its reward process through polymorphisms of the D2 dopamine receptor gene: a review, Eur. Psychiatry 15 (2000) 79-89.

[11] U. Ott, M. Reuter, J. Hennig, D. Vaitl, Evidence for a common biological basis of the absorption trait, hallucinogen effects, and positive symptoms: epistasis between 5-HT2a and COMT polymorphisms, Am. J. Med. Genet. B Neuropsychiatr. Genet. 137 (2005) 29-32.

[12] A.M. Persico, G. Bird, F.H. Gabbay, G.R. Uhl, D2 dopamine receptor gene TaqI A1 and B1 restriction fragment length polymorphisms: enhanced frequencies in psychostimulant-preferring polysubstance abusers, Biol. Psychiatry 40 (1996) 776-784.

[13] M. Reuter, J. Hennig, Pleiotropic effect of the TPH A779C polymorphism on nicotine dependence and personality, Am. J. Med. Genet. B Neuropsychiatr. Genet. 134 (2005) 20-24.

[14] J. Reuter, T. Raedler, M. Rose, I. Hand, J. Glascher, C. Büchel, Pathological gambling is linked to reduced activation of the mesolimbic reward system, Nat. Neurosci. 8 (2005) 147-148.

[15] M. Reuter, A. Schmitz, P. Corr, J. Hennig, Molecular genetics support Gray's personality theory: the interaction of COMT and DRD2 polymorphisms predicts the behavioural approach system, Int. J. Neuropsychopharmacol. (2006) 155-166.

[16] W. Schultz, Getting formal with dopamine and reward, Neuron 36 (2002) 241-263.

[17] A.H. Tran, R. Tamura, T. Uwano, T. Kobayashi, M. Katsuki, G. Matsumoto, T. Ono, Altered accumbens neural response to prediction of reward associated with place in dopamine D2 receptor knockout mice, Proc. Natl. Acad. Sci. U.S.A. 99 (2002) 8986-8991. 